\title{
Editorial
}

\section{Cholesterol Issues in Japan - Why Are the Goals of Cholesterol Levels Set So Low?}

\author{
Tomohito Hamazaki $^{a}$ Harumi Okuyama ${ }^{b}$ Yoichi Ogushi ${ }^{c}$ Rokuro Hama ${ }^{d}$ \\ ${ }^{a}$ Toyama Onsen Daini Hospital, Toyama City, ${ }^{b}$ Institute for Consumer Science and Human Life, Kinjo Gakuin \\ University, Moriyamaku, ${ }^{\mathrm{C}}$ Tokai University, Hiratsuka, and ${ }^{\mathrm{d}}$ Non-Profit Organization Japan Institute of \\ Pharmacovigilance (Kusuri-no-Check), Osaka, Japan
}

This summary aims to elucidate the relationship between serum total cholesterol (TC) or LDL-cholesterol (LDL-C) levels and mortality from all and cardiovascular causes in Japan, and to criticize the recently revised 'Japan Atherosclerosis Society (JAS) Guidelines for Prevention of Atherosclerotic Cardiovascular Diseases, 2012' [1] (JASG 2012). The hypothesis 'the lower the cholesterol levels are, the better' is absolutely wrong.

The epidemiological studies in Japan with $>10,000$ participants over follow-ups of $>10$ years showed that allcause mortality in the groups of the highest TC or LDL-C levels was lower than most of the other groups [2-4]. The only exceptional study (NIPPON DATA 80 , on which JASG 2012 depends almost exclusively) claimed that the group of the highest TC levels had the highest all-cause mortality [5]. However, this study had several important flaws. First, results were adjusted for sex and albumin levels. Without adjustment for sex, all-cause mortality in the groups of the highest TC levels ( $>259 \mathrm{mg} / \mathrm{dl})$ was not significantly higher than control groups (160-179 mg/dl) either in men or women. Serum albumin levels are positively associated with TC levels, especially in elderly people [6]. Thus, the adjustment for albumin (a negative risk factor for all-cause mortality) cancelled good aspects of TC. Second, the authors excluded liver disease deaths from all-cause mortality in one of their analyses claiming that liver disease caused both death and depression of cholesterol levels and that low cholesterol levels were not the cause of death [5]. Without liver disease death, this would be no longer all-cause mortality. Besides, hepatitis $\mathrm{C}$ virus (HCV) enters hepatic cells via LDL receptors [7], and low cholesterol levels are one of the major risk factors of $\mathrm{HCV}$ infection and chronic hepatitis. Hence, death from liver disease could be the result of low cholesterol levels [8]. Third, NIPPON DATA 80 apparently included a higher percentage of participants with familial hypercholesterolemia $(\mathrm{FH})$ than the general Japanese population [9], which exaggerated the risk of TC. With regard to Japanese women, no study has ever shown that TC or LDL-C is a risk factor of mortality from coronary heart disease (CHD) except for that flawed NIPPON DATA 80 [5]. Consequently, it is hard to understand why women for primary intervention comprise nearly two thirds of statin users in Japan [Ogushi, unpubl. data].

JASG 2012 has many flaws. It omitted many important pieces of information that the readers have to know. The strangest point is that the committee members of JASG 2012 did not disclose COI at all. How can one assess the reliability of medical guidelines without COI disclosure? In fact, most of the committee members are known to be recipients of huge amounts of research grants from the related industries. The following are some other unacceptable points of JASG 2012:

\section{KARGER \\ Fax +4161306 1234 \\ E-Mail karger@karger.ch \\ www.karger.com}

(C) 2012 S. Karger AG, Basel

0250-6807/13/0621-0032\$38.00/0

Accessible online at:

www.karger.com/anm
Dr. Tomohito Hamazaki

Toyama Onsen Daini Hospital

Taromaru-Nishimachi

Toyama City, Toyama 9338271 (Japan)

E-Mail hamazaki@inm.u-toyama.ac.jp 


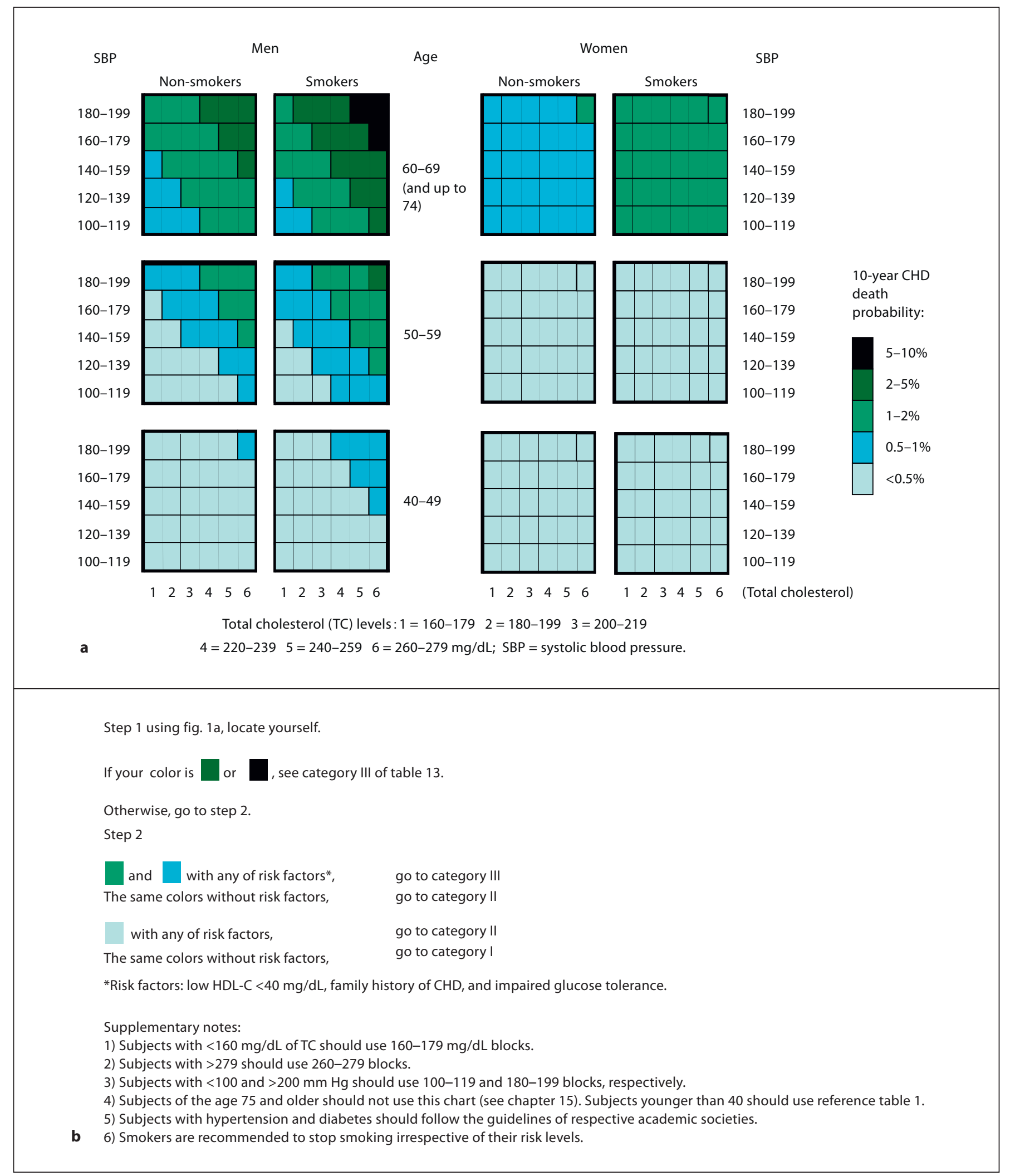

Fig. 1. The most important figure found in JASG 2012 (Fig. 7 in Ch. 4). Using this figure and Table 1 (next page), you can find your treatment goals of lipids. See notes in part $\mathbf{b}$ for details. 
Table 1. Table 13 in JAS-G 2012: treatment goals of lipids according to risk levels

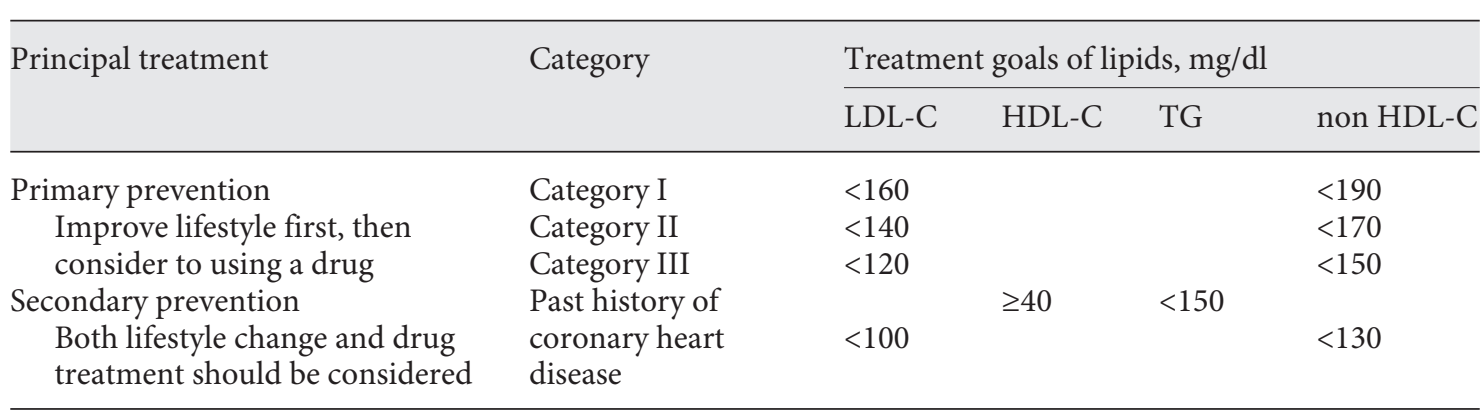

Supplementary notes:

1. Refer to Chapter 9 for familial hypercholesterolemia.

2. Refer to Chapter 15 for subjects 75 years of age and older.

3. Refer to Reference Table 1 for young subjects and other subjects with low risk.

4. The listed values above are only goals to strive for.

5. The goal for LDL-C may be set 20-30\% below the starting level.

6. The goal for non-HDL-C is the secondary goal after reaching the goal of LDL-C levels in patients with hypertriglyceridemia. Non-HDL-C should be used when triglyceride levels are $\geq 400 \mathrm{mg} / \mathrm{dl}$ or when blood is sampled after meal.

7. In any category, the principle to reach the goal is lifestyle improvement.

8. In category I, drugs are administered only when LDL-C levels are $\geq 180 \mathrm{mg} / \mathrm{dl}$.

Comments from the present authors: Chapters 9 and 15, and Reference Table 1 are not shown here. Diet and lifestyle improvement can decrease TC only a few percent. Thus, JASG 2012 practically does nothing but recommend drug use from the beginning.

(1) The relationship between TC or LDL-C and all-cause mortality was not described in JASG 2012. Without this information, all treatment guides for lowering cholesterol are just misleading. The group of people whose mortality is the lowest is the group which needs least treatment.

(2) The most important chart in JASG 2012 is probably shown in figure la and $b$ (figure 7 in Chapter 4 of JASG 2012). This chart depicts the absolute risk of CHD death according to the following risk factors: sex, smoking status, age, systolic blood pressure, and TC. For example, the men's chart has 180 boxes with five different colors indicating levels of CHD mortality. Because there are five risk levels (colors), there are four borders among five colors. According to the original paper of this chart (one of the NIPPON DATA 80 papers [10]) and our estimation, the number of male deaths used for the chart is around 45 . There are 180 risk level boxes. How can one draw four border lines among 180 boxes? This chart is purely the opinion of experts (or consensus as they say), and not based on statistical significance.

(3) In table 1 (table 13 in Chapter 4 of JASG 2012), treatment goals (LDL-C $<160 \mathrm{mg} / \mathrm{dl}$ ) are described even for women without any risk. Women's cholesterol levels must not be lowered, because even figure 7 in JASG 2012 could not show this (see the right half of fig. 1a).

(4) The original paper for figure 1 described the relationship between TC levels and the risk of death from stroke [10]. Although it might not be significant, it can be easily understood that TC was a negative risk factor for stroke. JASG 2012 does not include this information. Why do they have to omit this information?

(5) Figure 1 and table 1 appear to claim that the age risk factor increases the risk of high TC or LDL-C levels. No epidemiological studies have ever proved such a hypothesis. Actually the fact is the other way around. People before their 40 s or 50 s include surviving FH subjects and other similar genetically hypercholesterolemic people. It is highly likely that an inverse correlation between TC (or LDL-C) and all-cause mortality becomes prominent with age in the Japanese because the fraction of participants with $\mathrm{FH}$ becomes smaller with age [11]. Age is not the factor to lower the LDL-C goal levels.

(6)Non-HDL-cholesterol is newly introduced as a risk factor in table 1. In 1997, JAS published their first version of hyperlipidemia guidelines. In that version, they described that TC was the risk factor. After a few revisions they changed their target from TC to LDL-C in 
Table 2. Table 16 in JAS-G 2012: diet to prevent atherosclerotic disease

1 To maintain the standard BMI (22) considering energy intake and physical activity

2 To maintain the fat energy \% between 20 and 25\%, saturated fatty acids between 4.5 and $7 \%$, and cholesterol intake $<200 \mathrm{mg} /$ day

3 To increase $n-3$ polyunsaturated fatty acids

4 To maintain the carbohydrate energy \% between 50 and $60 \%$, and to increase dietary fibers

5 To set the goal of salt intake $<6 \mathrm{~g} /$ day

6 To maintain alcohol intake $<25 \mathrm{~g} /$ day

Comments from the present authors: Some of the above recommendations are hardly evidence-based.

2007. At that time, JAS did not have any Japanese data associating LDL-C with CHD at all. Now JAS claims non-HDL-cholesterol as another marker. This illustrates how weak their evidence about cholesterol is.

(7)JASG 2012 recommends that intake of saturated fatty acids should be no more than $7 \%$ of the total calories. No epidemiological studies in Japan have endorsed this recommendation. On the contrary, participants in the highest quintile of saturated fatty acid intake had the lowest mortality from stroke ( $\mathrm{p}$ for trend $=0.004$ ), and mortality from cardiovascular disease also had a similar trend $(\mathrm{p}=0.05)$ (JACC Study) [12]. JASG 2012 recommends that cholesterol intake should be $<200$ $\mathrm{mg} /$ day (table 2). No such evidence exists in Japan.

(8) The evidence of benefit by lowering TC or LDL-C is scarce in Japan. No large-scale RCT has been completed in Japan. The MEGA Study [13], which seems the major evidence for statin treatment in Japan, compared two kinds of intervention for patients with hypercholesterolemia: diet with pravastatin versus diet alone. Randomization of the MEGA Study was broken because significantly more participants were withdrawn in the pravastatin group, and around two thirds of participants dropped out of the study after they were asked to extend participation from 5 to 6 years (an unforgivable protocol violation). Unfortunately, participants in both groups were asked to take margarine (containing trans fatty acids or linoleic acid in large amounts) instead of butter and not to eat fatty fish. Because TC levels were open to all participants and doctors, participants in the active group, whose
TC levels were much more lowered than in the dietalone group, were less motivated to follow the wrong diet. This is probably one of the major reasons why the $\mathrm{CHD}$ incidence was lower in the active group (the absolute difference was less than $1 \%$ at $\mathrm{p}=0.01$ ). Very strange to say, the incidence of $\mathrm{CHD}$ in the active group was zero for over 1 year (such a rare thing happened at $p=0.01$, a very conservative estimate) just after the extension procedure (from 5 to 6 years) started whereas incidence accumulation continued at the same rate as before in the diet-alone group.

(9) The first large-scale intervention trials with simvastatin in Japan were performed for patients with TC values of $220-299 \mathrm{mg} / \mathrm{dl}$ (J-LIT) [14]. The results were cited as evidence to support the JASG 2012. However, mortality rates for $\mathrm{CHD}$, cancer and all causes increased along with decreasing TC from $220 \mathrm{mg} / \mathrm{dl}$ to lower levels. An area-matched control study of J-LIT, published in Japanese separately [15], showed the allcause mortality was greater in the statin-treated group than that of the control group.

(10) The reported adverse effects of statins include myopathy, carcinogenicity [16], disorder of the central and peripheral nervous systems [17], onsets of diabetes [18], and others [19]. However, comments on potential adverse effects associated with cholesterol-lowering drugs are very few. Actually no comments about the diabetogenic property of statins are found in JASG 2012.

As far as lipids go, JASG 2012 is based on very weak evidence, and essentially an expert's opinion with limited knowledge. In Japan, where CHD death rates are only one third of that in the United States, the very stringent goals for cholesterol levels do not make any sense at all. Most of our criticism to the JASG 2012 is applicable to other countries' guidelines, too.

References

1 Japan Atherosclerosis Society Guidelines (2012 Version) - Setting Committee Members. Japan Atherosclerosis Society (JAS) Guidelines for Prevention of Atherosclerotic Cardiovascular Diseases, 2012 (in Japanese). Tokyo, Japan Atherosclerosis Society, 2012.

$\checkmark 2$ Noda H, Iso H, Irie F, Sairenchi T, Ohtaka E, Ohta H: Gender difference of association between LDL cholesterol concentrations and mortality from coronary heart disease amongst Japanese: the Ibaraki Prefectural Health Study. J Intern Med 2010;267:576587. 
3 Ogushi Y, Kurita Y: Cohort study for general population to analyze relations between health check-up results and mortalities. Mumps (M Technology Association Japan) 2008;24:9-19.

-4 Nago N, Ishikawa S, Goto T, Kayaba K: Low cholesterol is associated with mortality from stroke, heart disease, and cancer: The Jichi Medical School Cohort Study. J Epidemiol 2011;21:67-74.

5 Okamura T, Tanaka H, Miyamatsu N, Hayakawa T, Kadowaki T, Kita Y, Nakamura Y, Okayama A, Ueshima H; NIPPON DATA 80 Research Group. The relationship between serum total cholesterol and all-cause or cause-specific mortality in a 17.3-year study of a Japanese cohort. Atherosclerosis. 2007; 190:216-223.

6 Ogushi Y: High cholesterol level is a good predictor of longevity in Japan. Proc IHEPA Int Conf 2011;3:1-8.

-7 Agnello V, Ábel G, Elfahal M, Knight GB, Zhang QX: Hepatitis C virus and other flaviviridae viruses enter cells via low density lipoprotein receptor. Proc Natl Acad Sci USA 1999;96:12766-12771.

8 Hama R: Low cholesterol and diseases: lipid rafts, LDL-HCV receptor and statin toxicity. 21st Annual Scientific Meeting of Japan Society for Lipid Nutrition, 2012.
>9 Hamazaki T, Okuyama H, Ogushi Y: Cholesterol-ronso - ueshima-shi no ronbun ni taisuru hanron (Cholesterol controversy - objection against Ueshima's paper) (in Japanese). J Lipid Nutr 2012;21:77-87. https:// www.jstage.jst.go.jp/article/jln/21/1/21_ 1_77/_pdf.

10 NIPPON DATA 80 Research Group: Risk assessment chart for death from cardiovascular disease based on a 19-year follow-up study of a Japanese representative population. Circ J 2006;70:1249-1255.

11 Okuyama H, Ichikawa Y, Sun Y, Hamazaki T, Lands WEM: Chapter 2 Association of high total cholesterol with coronary heart disease mortality differs among subject populations - familial hypercholesterolemia as a key concept. World Rev Nutr Diet 2007;96: 19-36.

12 Yamagishi K, Iso H, Yatsuya H, Tanabe N, Date C, Kikuchi S, Yamamoto A, Inaba Y, Tamakoshi A; JACC Study Group: Dietary intake of saturated fatty acids and mortality from cardiovascular disease in Japanese: the Japan Collaborative Cohort Study for Evaluation of Cancer Risk (JACC) Study. Am J Clin Nutr 2010;92:759-765.

13 Nakamura H, Arakawa K, Itakura H, Kitabatake A, Goto Y, Toyota T, Nakaya N, Nishimoto S, Muranaka M, Yamamoto A, Mizuno K, Ohashi Y; MEGA Study Group: Primary prevention of cardiovascular disease with pravastatin in Japan (MEGA Study): a prospective randomised controlled trial. Lancet 2006;368:1155-1163.
14 Matsuzaki M, Kita T, Mabuchi H, Matsuzawa Y, Nakaya N, Oikawa S, Saito Y, Sasaki J, Shimamoto K, Itakura H; J-LIT Study Group: Japan Lipid Intervention Trial. Large-scale cohort study of the relationship between serum cholesterol concentration and coronary events with low-dose simvastatin therapy in Japanese patients with hypercholesterolemia. Circ J 2002;66:1087-1095.

15 Yoshiike N, Tanaka H: What we have learnt from large-scale epidemiological studies performed in Japan. Area-matched control study for Japan Lipid Intervention Trial (JLIT). Lipid 2001;12:281-289.

16 Ravnskov U: Correspondence. JNCI 2008; 100:972-973.

17 Statins: Updated product information in patient leaflets on adverse reactions. Drug Safety Update 2009;3:11. http://www.mhra. gov.uk/home/groups/pl-p/documents/publication/con062549.pdf.

18 Culver AL, Ockene IS, Balasubramanian R, Olendzki BC, Sepavich DM, WactawskiWende J, Manson JE, Qiao Y, Liu S, Merriam PA, Rahilly-Tierny C, Thomas F, Berger JS, Ockene JK, Curb JD, Ma Y: Statin use and risk of diabetes mellitus in postmenopausal women in the Women's Health Initiative. Arch Intern Med 2012;172:144-52.

19 Goldstein MR, Mascitelli L, Pezzetta F: The double-edged sword of statin immunomodulation. Int J Cardiol 2009;135:128-130. 